\title{
An Environmental Monitoring System with Integrated Wired and Wireless Sensors
}

\author{
Jue Yang, Chengyang Zhang, Xinrong Li, Yan Huang, \\ Shengli Fu, and Miguel Acevedo \\ University of North Texas, Denton, Texas 76207, USA \\ $\{$ jy0074, cz0022, xinrong, huangyan, fu, acevedo\}@unt.edu
}

\begin{abstract}
Wireless sensor networks (WSN) technology has great potential to revolutionize many science and engineering domains. We present a novel environmental monitoring system with a focus on the overall system architecture for seamless integration of wired and wireless sensors for long-term, remote, and near-real-time monitoring. We also present a unified framework for sensor data collection, management, visualization, dissemination, and exchange, conforming to the new Sensor Web Enablement standard. Some initial field testing results are also presented.
\end{abstract}

\section{Introduction}

Environmental monitoring applications have become major driving forces for wireless sensor networks (WSN) 12]. Ecological and environmental scientists have been developing a cyber infrastructure in the form of environmental observatories, consisting of a variety of sensor systems, sophisticated computational resources and informatics, to observe, model, predict, and ultimately help preserve the health of the natural environment. Such an infrastructure becomes more important as we recognize that the natural world is inextricably linked to the human society to form an extremely complex ecosystem. WSN-based environmental monitoring systems promise to enable domain scientists to work with data sets of unprecedented fine spatial and temporal resolution.

In this paper, we present a novel environmental monitoring cyber infrastructure that features (1) soil moisture monitoring with flexible spatial coverage and resolution, (2) seamlessly integrated wired and wireless sensors, (3) long-term autonomous remote near-real-time monitoring, (4) publicly available web services for sensor data visualization and dissemination, and (5) remote system monitoring and maintenance. Despite significant advances in recent years, there are still many challenging issues to be addressed to fulfill the full potential of the emerging WSN technology. The importance of empirical study of WSN has been widely recognized by the research community and considerable efforts have been put into the development and deployment of WSN testbed for various practical applications, including environmental monitoring [1,2,3,4/5,6/7,8]. However, there are many limitations in the existing WSN testbed deployments. For example, many deployments are in controlled environments, instead of real-life 
application environments. Most of the deployments are designed for short-term experiments or proof-of-concept demonstration purposes, instead of long-term autonomous operation to support ongoing work by domain scientists and practitioners. Most of the deployments are stand-alone WSN-only system, monitoring very few environmental parameters, instead of being a part of the ever-growing environmental monitoring cyber infrastructure. As a result, it is difficult to consolidate a broad range of sensor data systematically to study the cross-correlation among various environmental parameters.

In this research, we intend to fill the aforementioned gaps in WSN research by developing a soil moisture monitoring WSN system and integrating it into the Texas Environmental Observatory (TEO) infrastructure [9] for long-term operation. The new WSN-based soil moisture monitoring system will mainly support long-term hydrologic monitoring and modeling research. Increasing urbanization brings changes to the land cover of a given drainage area, which in turn increase the quantity of water flowing overland and decrease the amount of time to reach peak flow [10], increasing in some cases the risk of flash floods. Hydrologic models are helpful in predicting how changes in land cover in rapidly urbanizing areas translate into changes in the stream flow regime. These models require inputs that are difficult to measure over large areas, especially variables related to storm events, such as soil moisture antecedent conditions and rainfall amount and intensity. The new monitoring system that we are developing is ideally suited for such applications.

The rest of the paper is organized as follows. In Section 2, we identify key design requirements for the new environmental monitoring system. The overall system architecture is described in Section 3. Then, in the next three sections, we describe three major components of the new system, including WSN for soil moisture monitoring, gateway and telemetry system for remote near-real-time environmental monitoring, and web services for data visualization and dissemination. In Section 7, some initial field test results are presented. At last, the paper is closed with a summary and future work in Section 8

\section{Design Requirements}

In this research, we develop a new environmental monitoring system to significantly improve the capability and usability of the system that is currently deployed at the Greenbelt Corridor (GBC) Park, operated by Texas Parks and Wildlife Departments, Denton, Texas. Some key design requirements are identified in this section.

Soil moisture monitoring with flexible spatial coverage and resolution: In the existing system, all sensors are deployed inside a small fence-enclosed area, a situation typical of many environmental monitoring systems. There is a need to provide flexibility to extend the spatial coverage and adjust the spatial resolution of soil moisture sensors. The spatial coverage of the system is limited by the physical limitation on the length of the cable connecting the sensors to 
the datalogger. In contrast, the spatial coverage and resolution of WSN can be conveniently configured to be meaningful to domain scientists.

Integration of WSN with existing environmental observatories: Despite its limitations, traditional environmental monitoring systems with various wired sensors are capable of accomplishing many monitoring tasks, and substantial investments are in place to monitor temperature, wind speed and direction, rainfall, and solar radiation. Drastically replacing existing systems with an immature technology such as WSN is considered unacceptable to many domain scientists and practitioners. Therefore, it is important to introduce the new WSN technology without disrupting ongoing operation of environmental observatories through seamless integration of wired and wireless sensors.

Long-term, autonomous, remote, near-real-time environmental monitoring: It has been recently recognized that many ecological and environmental studies should implement long-term data collection and management. In addition, many environmental observatories are deployed in remote areas that are inconvenient to access for data retrieval and system deployment and management. Thus, environmental monitoring systems need to be survivable in extreme environmental and weather conditions for long-term operation with limited human intervention, which makes energy harvesting and energy efficiency major design considerations. Near-real-time sensor data collection is another important feature of monitoring systems to support time-sensitive environmental studies, which necessitates a convenient yet reliable long-haul wireless link.

Publicly available web services for sensor data visualization and dissemination: It is important to make data publicly available to benefit a broad range of entities such as environmental researchers, local citizens and government policy makers, and K-12 teachers and students. In addition, explosive growth of environmental data collected by a variety of sensors in long-term operation necessitates a unified framework for data collection, management, integration, visualization, and dissemination. Such a framework should conform to standards, such as the Sensor Web Enablement (SWE) standard proposed by the Open Geographic Consortium (OGC) [1], to enable data exchangeability and interoperability.

Remote system status monitoring and management: For environmental monitoring systems deployed in remote areas, remote monitoring of system status is extremely useful for system development, debugging, and maintenance purposes. Thus, various system status data need to be carefully defined and collected together with environmental sensor data. Furthermore, it is important to remotely adjust system configurations, update and upgrade software programs.

\section{Overall System Architecture}

The new environmental monitoring system can be divided into four major layers as shown in Fig. 1, including physical data layer, logical data layer, web presentation layer, and user layer. Such a layered approach makes it possible to implement the system in a flexible, extensible, and efficient way. At the physical data layer, a variety of sensors are employed to monitor environmental parameters. Sensor 


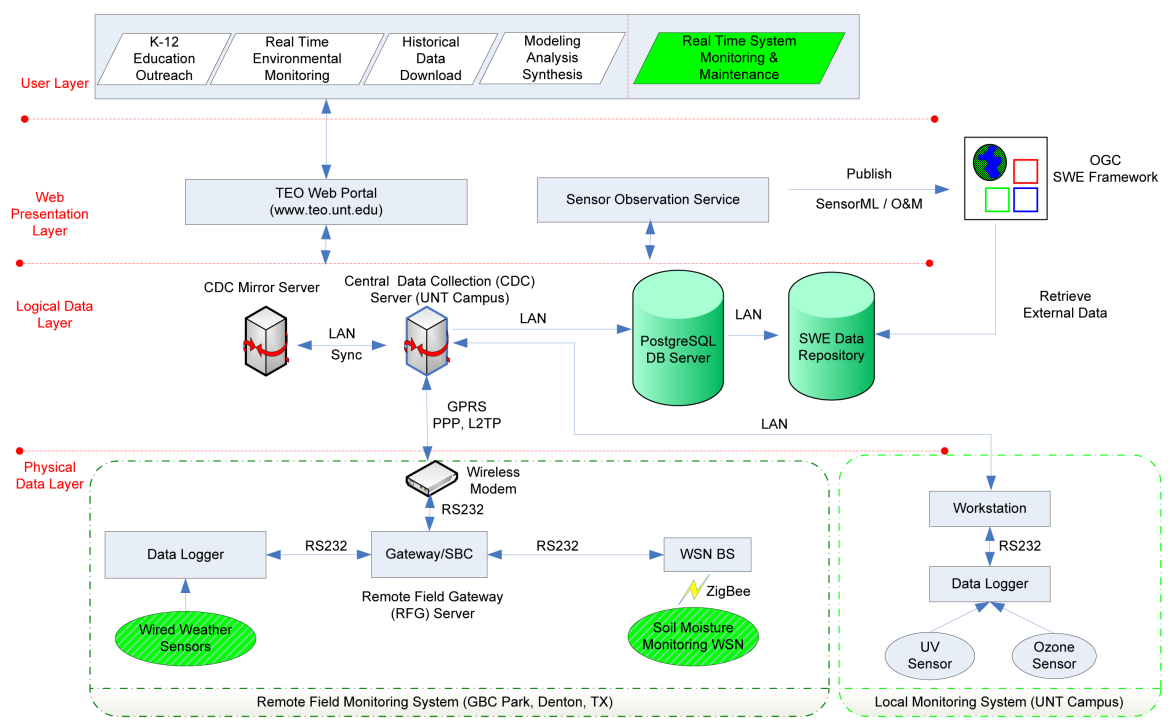

Fig. 1. System architecture of the integrated environmental monitoring system

data are transmitted from monitoring site to a Central Data Collection (CDC) Server. To address the design requirements, we incorporate a GPRS modem for wireless telemetry, a single-board computer (SBC) operating as a Remote Field Gateway (RFG) Server, and WSN for distributed soil moisture monitoring. The RFG Server provides effective control, management, and coordination of two relatively independent sensor systems, i.e., a traditional datalogger-based wired sensor system and the WSN-based wireless sensor system. The Linux-based RFG Server also supports remote login to allow maximum remote manipulation of the devices in the field such as the SBC, datalogger, and WSN.

At the logical data layer, sensor data collected from distributed monitoring stations are stored in a PostgreSQL Database (DB) Server. The CDC Server acts as an intermediate component to hide the heterogeneity of different physical layer devices and support data validation required by the DB Server. The CDC Server and its mirror server also archive raw data on local file systems. Daemon programs running on the CDC Server pre-process the data before it is inserted into the database, and periodically perform synchronization tasks. A SWE-compliant data repository is installed to enable data exchange, accepting data from both internal DB Server and external sources through the OGC web services.

The web presentation layer consists of a web portal, TEO Online [9], and a sensor web implementation. The web portal serves as a user-friendly interface to perform data visualization, analysis, synthesis, modeling, and K-12 educational outreach activities. It also provides useful capabilities for system developers and operators to remotely monitor system status and remotely update software and system configuration, which greatly simplifies system debugging and maintenance 
tasks. We also implement Sensor Observation Services (SOS) at this layer, conforming to the SWE standard to facilitate data exchange. The standard Sensor $\mathrm{ML} / \mathrm{O} \& \mathrm{M}$ data representation makes it easy to integrate our sensor data into the existing Geographic Information Systems (GIS) web services and exchange the data with many other organizations. The SOS web service will be published to a catalog service in the OGC SWE framework to make it publicly accessible on the Internet.

Finally, the user layer abstracts a variety of needs for education, outreach, research, and system development and management purposes.

\section{Wireless Sensor Networks for Soil Moisture Monitoring}

The WSN hardware platform employed in our current design is the MICAz mote from Crossbow 12. The MICAz mote provides a highly integrated, cost-effective hardware solution for low-power WSN applications. At each sensor node, a soil moisture sensor probe is connected to an optional MDA300 data acquisition board. The base station (BS) node is installed on an extension board MIB510, which interfaces with the RFG Server through RS232 serial ports. Data collected by each sensor node are periodically transmitted to the BS node through multihop communications. Then, BS transmits aggregated data package to the RFG Server through RS232 serial communication.

To accomplish long-term operation with minimum human intervention, motes are powered by solar cells and rechargeable batteries. The capacities of the rechargeable battery and solar cell are determined through power budget analysis of the system (see Section 5 for more details of such analysis). To survive extreme weather conditions, motes are packaged in weatherproof boxes and the boxes are installed 4 feet above the ground on top of metal poles to avoid flooding water and prevent fallen leaves from covering solar cells.

In general, in environmental monitoring applications, every sensor node needs to periodically carry out three main tasks as shown in Fig. 2 including data generation through sensing, data processing, and data reporting through multihop wireless communications. To accomplish the data generation task, sensor readings are collected periodically, at certain desired frequency, and sensor data are time-stamped upon sampling, which necessitates global time synchronization in the network. Then, to perform data processing task, sensor nodes calibrate, aggregate, summarize, and compress data. For example, complex data aggregation and summarization techniques such as the E2K method proposed in [13] may be employed to reduce wireless communications by exploiting the spatial and temporal correlation properties that are inherent in many sensor data. Lastly, during the data reporting task, sensor data are transmitted to the BS node through multihop wireless communication. The data reporting task is enabled by a variety of software services as shown in Fig. 2, which implements essential timing, communication, and networking protocols for energy-efficient multihop sensor data collection in distributed networks. 


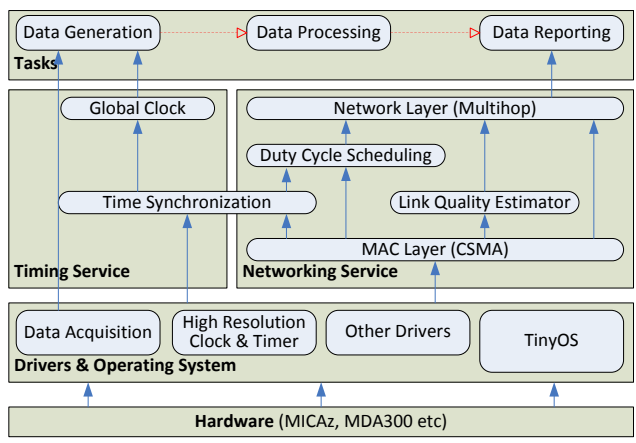

Fig. 2. Functional block diagram of sensor node

As discussed in Section 2, energy efficiency is one of the major design considerations. In wireless networks, Media Access Control (MAC) layer protocols are broadly categorized into two groups: schedule-based and contention-based methods 14. In schedule-based protocols such as time, frequency, and code division multiple access methods, wireless devices are scheduled to occupy different channels that are physically or logically independent. In contrast, in contentionbased protocols such as the carrier-sense multiple access (CSMA) method, wireless devices compete for a single shared channel. A schedule-based protocol could completely eliminate collision in wireless communication but it may not be able to adapt to the rapidly changing channel conditions whereas a contention-based protocol is highly autonomous but relatively energy-inefficient due to high collision rate in the shared channel. In this research, we develop a hybrid MAC layer protocol that integrates CSMA and duty-cycle scheduling. The hybrid protocol employs a distributed duty-cycle scheduling algorithm to coordinate sensor nodes' sleeping. The duty cycle of each node is scheduled by its parent. Sensor nodes remain asleep until their own reporting period; during reporting period, CSMA is employed to avoid any unexpected collision due to overlapping reporting periods of different sub-trees. Thus, the hybrid protocol strives to retain the flexibility of contention-based protocols while improving energy efficiency in multihop networks. Such a design is similar to the S-MAC protocol proposed in [14. However, S-MAC does not maintain a network-wise global time so that sensors must adopt schedules from multiple virtual clusters.

The performance of schedule-based methods depends on the accuracy of time synchronization. The flooding time synchronization protocol (FTSP) proposed in 15. time-stamps synchronization messages at the MAC layer, which removes the non-deterministic delay at both sender and receiver caused by uncertain processing time in the operating system for context switches, system call overhead, interrupt handling, and other essential operations. FTSP is able to synchronize multiple receivers with a single broadcast message. Such a floodingbased method is also insensitive to topological changes. In environmental monitoring applications, distributed sensor nodes typically form a spanning-tree 
structure, rooted in a single data-collection BS node serving as a gateway to the remote monitoring and control center. In this research, a modified FTSP is developed to better suit environmental monitoring applications by exploiting the unique tree-structured network architecture. In a tree-structured network, every node synchronizes to its parent node and thus ultimately all nodes synchronize to the root node to achieve global time synchronization in the network.

The multihop routing protocol at the network layer is responsible for establishing and keeping up the routing hierarchy in distributed WSN. While the routing structure in environmental monitoring applications is a simple tree and the data flow is almost one-directional, the dynamic and unreliable nature of the wireless communication poses great difficulty in organizing and maintaining a reliable multihop routing hierarchy. In this research, we implement an exponential weighted moving average (EWMA) estimator-based link quality estimator [16. The weight used here is the normalized received signal strength of the synchronization packet, which will then be halved in each cycle. Such an estimator reacts quickly to potentially large changes in link quality yet is stable enough when changes are small. The multihop routing protocol makes use of the link quality estimate to maintain a reliable routing topology.

\section{$5 \quad$ Wireless Telemetry System}

To seamlessly integrate a variety of devices in the field, as shown in Fig. 1] we implement a RFG Server using a rugged ultra-low-power SBC TS-7260 from Technology Systems, Inc [17. To minimize energy consumption, the monitoring system is automatically switched between active and sleep modes. The sleep mode of the SBC is enabled by the optional battery backup board TS-BAT3, which also serves as an embedded uninterruptible power supply (UPS). The devices deployed in the field are commonly equipped with RS232 serial port, including data loggers, wireless modem, and WSN BS node. Thus, with five serial ports onboard, the SBC is well suited to serve as gateway server.

The long-haul wireless link from the field to the CDC Server is implemented using a GPRS modem. The GPRS, standing for General Packet Radio Service, is a packet oriented mobile data service available to the subscribers of GSM cellular networks. The GPRS link is maintained by the SBC, using Point-toPoint (PPP) protocol, a data link protocol commonly used to establish a direct connection between two nodes over serial cable, phone line, cellular phone, or dial-up network. Once the SBC boots up, it will automatically dial to GPRS network, and keep the link alive during the entire active period. To enable secure system access, the Layer 2 Tunneling Protocol (L2TP) is used to support virtual private network that establishes a secure point-to-point connection between the RFG Server and the CDC Server through the public Internet. To be energyefficient, wireless modem is powered off during the system's sleep period.

Wireless telemetry system in the field is powered by solar energy with a large solar panel and rechargeable battery. The required capacities of rechargeable battery and solar panel are determined through power budget analysis. In such 
analysis, average power consumption of each power load device is determined by measuring or estimating the average current draw and the time spent in each of its operating modes. In practice, a lead-acid battery cannot be $100 \%$ discharged repeatedly. Therefore, it is necessary to de-rate the battery by some amount, generally 25\% [18]. To survive extreme weather conditions in long-term operation, we target at supporting the system with a fully charged battery for at least a week without recharging. In general, the capacity of a solar panel should be 10 times the average power consumption of the load [18].

The CDC Server receives data through either pull or push operations. In a pull operation, the CDC Server periodically connects to the data source and pulls the data. In a push operation, the CDC Server opens a port, such as File Transfer Protocol (FTP) port, through which the data can be pushed by the data source. Such a mechanism allows the CDC Server to flexibly adapt to different types of data sources. The sensor data collected by the CDC Server is first archived in the local file system. For each data source, a back-end data handler (daemon program) is used to check the integrity of the data. Based on a set of predefined validation rules, the data are cleaned up before sending to the PostgreSQL DB Server. Data handler may also require the RFG Server to recollect and retransmit missing data packets. Data handlers are running on separate user spaces to avoid conflict among different data sources. A new data handler is added for each new data source with minimal change in the database and web visualization layers. Therefore, the system scalability and extensibility are greatly enhanced.

Emphasis on system extensibility drives sensor database design because of the need to handle large volume of sensor data collected from heterogeneous sources in long-term operation. All sensor information is contained in one relation or table, while each observation is stored in a separate relation. When more sensors are deployed as the system expands, they are registered as new records in the sensor relation by data handlers on the CDC Server. Observations from new sensors are added as new tables. The observations and sensors are linked through unique sensor identification codes. Thus, they will be automatically recognized by the upper layer web applications once being added to the database. Such a design allows flexible system development and web interface design. It also facilitates conversion of the data to SensorML [19] to enable sensor data exchangeability and interoperability through web services.

\section{Sensor Data Visualization and Dissemination}

As an integral part of the new environmental monitoring system, we have developed a dedicated web portal, TEO Online [9, with a set of publicly available web services for sensor data visualization and dissemination. In designing data visualization framework, we take full advantage of the flexible Google Maps API facility to associate sensors with their geographical locations intuitively on the satellite-view map interface. Each sensor is represented by a KML (Keyhole Markup Language [20]) placemark and displayed as an interactive marker that links to a drilldown information page for detailed observation charts. Such an 


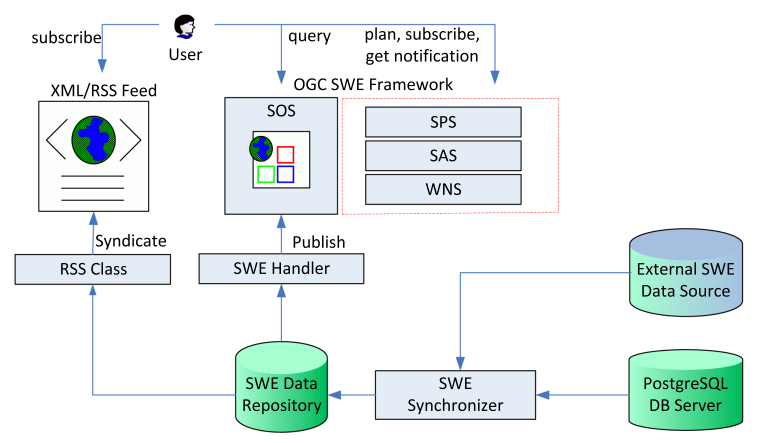

Fig. 3. Sensor data dissemination and exchange framework

interface provides direct visualization of spatial distribution of sensors and sensors data, which is an important aspect of environmental studies.

Real-time and historical sensor data as well as their temporal variation statistics may be viewed in several different formats. Depending on the type of natural phenomenon, various types of single and multi-series charts are provided. For a single series chart, users can adjust the data reporting interval and the zooming level of time range to view data trends at different temporal granularities. Several predefined functions allow the analysis of data statistics such as average and maximum values. With a multi-series chart, users can compare readings reported from sensors deployed at different locations to analyze spatial distribution characteristics of natural phenomena.

In this research, we develop a flexible framework to share sensor data with other parties through Extensible Markup Language (XML) data exchange, Really Simple Syndication (RSS) feed, and Sensor Observation Services (SOS) as shown in Fig. 3. The sensor data exchangeability is achieved by a dedicated SWE data repository as well as web services built upon the repository. RSS feed is a format that has been widely used to publish frequently changing data and allow users to subscribe such data. In our system, the RSS feed items and links are stored in an RSS table in the repository, while the live data is encapsulated in the RSS page by web layer RSS class functions. We also provide a more powerful data exchange interface through the OGC's SWE framework. SWE is a new standard that specifies interoperability interfaces and metadata encodings to enable realtime integration of heterogeneous sensor data [1]. The major encoding standards include SensorML that describes sensor system information, and O\&M (Observations and Measurements) that encode actual live observations [19. The interoperability interface standards include SOS (Sensor Observation Services) that allows near real-time retrieval of sensor data, SAS (Sensor Alert Services) that allow publish and subscription of the data, SPS (Sensor Planning Services) and WNS (Web Notification Services).

Our database is syndicated using sensor table, which automatically enables the conformation to the SWE standard. Thus, the SWE data repository can be easily synchronized with the DB Server through a SWE synchronizer. Meanwhile, 

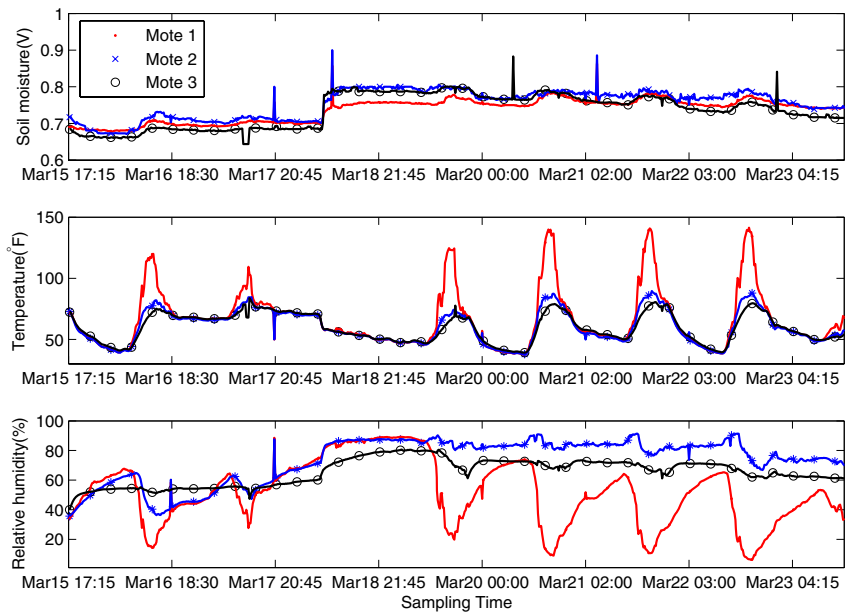

Fig. 4. Sample sensor data collected in field testing

the SWE synchronizer can also retrieve external data from any SWE-compatible data sources and confederate our repository. The data in the SWE repository is converted to SensorML and O\&M format by an SWE handler, which then feed the information to the upper layer web services.

\section{System Deployment and Field Testing Results}

The GBC weather and soil moisture station in Denton, Texas, has been operational for nine years with temperature, solar radiation, rain gauge, wind speed and direction, and soil moisture sensors, all connected to dataloggers. In March 2008, we expanded the GBC station by deploying a wireless modem, a SBC, and a pilot WSN to implement the integrated system shown in Fig. 1. In the current setup, the RFG Server wakes up every 15 minutes for data collection with a duty cycle of about $12 \%$. The wireless modem is powered-off during inactive periods. Eight motes are deployed in the surrounding area of the station and each mote collects data from a soil moisture sensor (connected by wire to the mote), and onboard temperature and relative humidity sensors. Motes wake up every 5 minutes for time synchronization and networking operations, but transmit sensor data only every 15 minutes following the same schedule as the RFG Server. The duty cycle of motes is about 1\%. Soil moisture data is kept in raw format while other data are converted into engineering units before transmission.

Figure 4 shows sample data collected by three motes during field testing. On March 18, GBC area experienced heavy rainfall and a significant drop of temperature. The rain event was monitored by a rain gauge connected to datalogger. The variation of weather condition was also captured by the sensors on motes as shown in Fig. 4. The soil moisture exhibits a jump on that day while temperature 
was falling. The difference among three soil moisture sensors' data reveals spatial variation characteristics of the soil moisture condition in that area, which is an invaluable input to the hydrologic modeling research. As a part of the field testing shown in Fig. 4, we installed motes in two different boxes to test packaging alternatives. Mote 1 is installed in a transparent box to put a solar cell inside the box while Mote 2 and Mote 3 are installed in non-transparent boxes with the solar cell installed outside the box. From the results, we can observe that Mote 1 experiences significantly higher temperature during the day than the other two as a result of the greenhouse effect inside the transparent box, which also results in much lower relative humidity for Mote 1 . The temperature may become very high in Texas in the summer. MICAz mote is designed to operate in harsh environment, but the rechargeable NiMh batteries cannot tolerate high temperature. Thus, we are currently evaluating more packaging options for survivable long-term deployment in the field.

The web portal 9] has been operational since March 2008 with most of the basic web services implemented. Various environmental sensor data and system status data from several monitoring sites can be viewed and downloaded from the web portal. Currently, the sensor data are being shared with the City of Denton and the National Weather Service in Fort Worth for various monitoring, modeling, and prediction purposes, which is made extremely convenient by the new data exchange framework described in Section 6.

\section{Summary and Future Work}

A remote near-real-time environmental monitoring system that is developed to support long-term environmental studies is presented in this paper with a focus on the overall system architecture for seamless integration of the emerging WSNbased system and the legacy wired sensor system. A unified framework for sensor data collection, management, dissemination, and exchange is also presented. Due to space limitation, many technical details of the system components are not covered in this paper; more design and implementation details of the soil moisture monitoring WSN and web services for sensor data visualization, management, dissemination, and exchange will appear in separate publications.

Currently, with support from the National Science Foundation (NSF), we are in the early stage of scaling-up the soil moisture monitoring WSN at the GBC site to around 100 motes to have a much larger geographic coverage than the current eight-mote deployment [9]. In addition, in cooperation with the City of Denton, the current remote monitoring system with WSN and wireless telemetry will be replicated at five other weather stations across the North Texas area. We are also deploying a large-scale WSN of around 100 motes in the GBC site to serve as an open research infrastructure for the WSN research community. To fulfill this goal, secure web services will be developed for remote over-the-air programming and configuration of WSN. Access to such web services will be provided to interested researchers who may carry out experimental studies of WSN from thousands of miles away. 


\section{Acknowledgments}

This research is supported in part by NSF under Grants OCI-0636421, CNS0709285, and EEC-0431818, and by Texas Advanced Research Program under Grant 003594-0010-2006. The authors would like to thank David Hunter (City of Denton), Sonny Solis (Texas Parks and Wildlife), Bob Carle (National Weather Service), and members of the TEO Research Group at UNT, especially Rudi Thompson (Biology), Duane Gustavus (CITC), Adam Skelton (EE MS student), Sanjaya Gurung (EE MS student), and Liping Chen (Applied Geography MS student) for their contributions to the development of the TEO system.

\section{References}

1. Culler, D., Estrin, D., Srivastava, M.: Overview of sensor networks. IEEE Computer, 41-49 (August 2004)

2. Martinez, K., Hart, J.K., Ong, R.: Environmental sensor networks. IEEE Computer, 50-56 (August 2004)

3. Mainwaring, A., Polastre, J., Szewczyk, R., Culler, D., Anderson, J.: Wireless sensor networks for habitat monitoring. In: Proc. of the ACM Int'l Workshop on Wireless Sensor Networks and Applications (WSNA) (September 2002)

4. MoteLab: Harvard Sensor Network Testbed, http://motelab.eecs.harvard.edu

5. ExScal: Extreme Scale Wireless Sensor Networking, http://cast.cse.ohio-state.edu/exscal

6. Kansei: Sensor Testbed for At-Scale Experiments, http://ceti.cse.ohio-state.edu/kansei

7. CitySense: An Open, Urban-Scale Sensor Network Testbed, http://www.citysense.net

8. SCADDS: Scalable Coordination Architectures for Deeply Distributed Systems, http://www.isi.edu/scadds/testbeds

9. Texas Environmental Observatory, http://www.teo.unt.edu

10. Cheng, S., Wang, R.: An approach for evaluating the hydrological effects of urbanization and its application. Hydrological Processes 16, 1403-1418 (2002)

11. Sensor Web Enablement WG, OGC Inc., http://www.opengeospatial.org

12. Crossbow Inc., http://www.xbow.com

13. Harrington, B., Huang, Y.: A two round reporting approach to energy efficient interpolation of sensor fields. In: Papadias, D., Zhang, D., Kollios, G. (eds.) SSTD 2007. LNCS, vol. 4605, pp. 130-147. Springer, Heidelberg (2007)

14. Ye, W., Heidemann, J., Estrin, D.: An energy-efficient MAC protocol for wireless sensor networks. In: Proc. of the 21st Int'l Annual Joint Conference of the IEEE Computer and Communications Societies (INFOCOM), vol. 3, pp. 1567-1576 (June 2002)

15. Maroti, M., Kusy, B., Simon, G., Ledeczi, A.: The flooding time synchronization protocol. In: Proc. of the ACM Conference on Embedded Networked Sensor Systems (SenSys) (November 2004) 
16. Woo, A., Tong, T., Culler, D.: Taming the underlying challenges of reliable multihop routing in sensor networks. In: Proc. of the Int'l Conference on Embedded Networked Sensor Systems (SenSys) (November 2003)

17. Technologic Systems Inc., http://www. embeddedarm.com

18. NovaLynx Corporation, Power Budget Calculations, http://www.novalynx.com

19. SensorML, UAH VAST, http://vast.uah.edu/SensorML

20. Keyhole Markup Language, Google, http://code.google.com/apis/kml 